\title{
Progressive Deconstruction of a Distal Posterior Cerebral Artery Aneurysm Using Competitive Flow Diversion
}

\author{
Andrew K. Johnson, MD', Lee A. Tan, MD², Demetrius K. Lopes, MD², \\ Roham Moftakhar, MD
}

Progressive deconstruction is an endovascular technique for aneurysm treatment that utilizes flow diverting stents to promote progressive thrombosis by diverting blood flow away from the aneurysm's parent vessel. While the aneurysm thromboses, collateral blood vessels develop over time to avoid infarction that can often accompany acute parent vessel occlusion. We report a 37-year-old woman with a left distal posterior cerebral artery aneurysm that was successfully treated with this strategy. The concept and rationale of progressive deconstruction are discussed in detail.

Key Words: Stents; Intracranial aneurysm; Subarachnoid hemorrhage

Distal posterior cerebral artery (PCA) aneurysms are rare and treatment can be challenging. "With aneurysms of the P2 and P3 segments," an expert vascular neurosurgeon has stated, "I prefer endovascular solutions whenever possible, given the surgical complexity"[1]. Lesions in this region may also be difficult to treat with endovascular methods because of

${ }^{1}$ Wellstar Neurosciences Network, Wellstar Health System, Kennestone Hospital, Marietta, Georgia, USA

${ }^{2}$ Department of Neurosurgery, Rush University Medical Center, Chicago, Illinois, USA

${ }^{3}$ Palmetto Health Neurosurgery Associates, Columbia, South Carolina, USA

Received December 7, 2015;

accepted after revision January 21, 2016.

Correspondence to: Lee A. Tan, MD, Department of Neurosurgery, Rush University Medical Center, 1725 West Harrison St \#855 Chicago, IL-60612, USA

Tel.312.942.6644 E-mail: lee_tan@rush.edu

This is an Open Access article distributed under the terms of the Creative Commons Attribution Non-Commercial License (http://creativecommons.org/licenses/by-nc/3.0) which permits unrestricted non-commercial use, distribution, and reproduction in any medium, provided the original work is properly cited. their distal location and the often-narrow caliber. Treatment has often resorted to parent vessel sacrifice when reconstructive strategies have not been successful. An experienced open and endovascular team still had $25 \%$ permanent morbidity/mortality with complex aneurysms in this region [2].

Progressive deconstruction has recently been described, wherein a flow diverter is placed across the origin of an aneurysm's parent vessel [3]. This deconstructive aneurysm treatment technique gradually thromboses both the aneurysm and the parent vessel, allowing adequate collateralization of the vasculature to prevent infarction. We report a case of progressive deconstruction of a complex distal PCA aneurysm.

\section{CASE REPORT}

A 37-year-old woman presented with new onset of headache and diplopia. She had no significant past medical history. Her neurological exam was otherwise unremarkable. Computed tomographic angiography of the brain revealed an unruptured left PCA aneurysm 
measuring $2.1 \times 2.0 \mathrm{~cm}$. The aneurysm was situated at the PCA bifurcation with its neck predominately involving the parieto-occipital artery (Fig. 1A, B).

After thorough discussion regarding management options, the patient elected to undergo endovascular treatment. The aneurysm was embolized with a portion of coil protruding into the PCA proximal to the aneurysm. She did not have any new neurological deficit after the procedure and was discharged in stable condition. The patient returned to the emergency department two weeks after the coiling procedure with a sudden onset of severe headache, nausea and vomiting. She did not have any focal neurological deficit on exam. Computed tomography (CT) and magnetic resonance imaging (MRI) demonstrated subarachnoid hemorrhage. She was again taken to the angiography suite for repeat coiling of the now ruptured left distal PCA aneurysm. The procedure was completed without complication with Raymond class II occlusion (small residual neck filling). The patient had a stable course in the hospital and eventually was discharged without neurological deficit.

Follow-up diagnostic cerebral angiography was obtained at six months after the repeat coiling

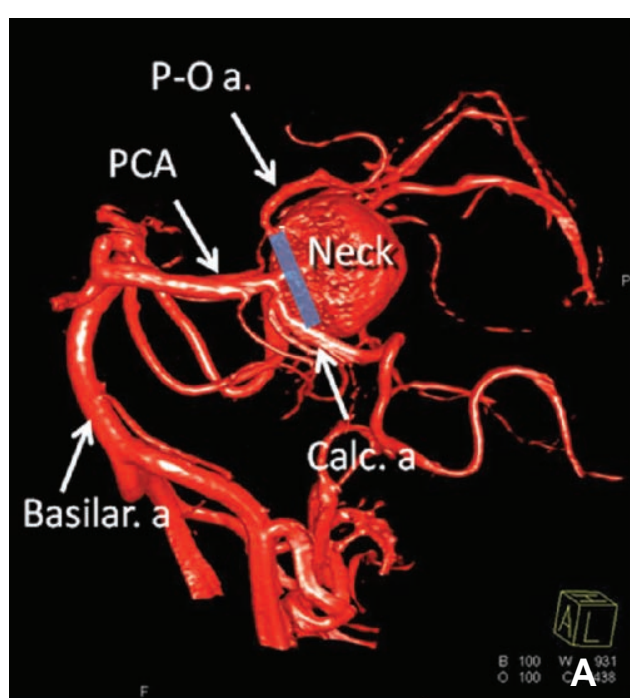

Fig. 1. A. Three-dimensional rotational angiography of a left vertebral artery injection showing a very large left posterior cerebral artery (PCA) bifurcation aneurysm prior to treatment; (B) cerebral angiograms after initial coiling; (C) cerebral angiogram after second coiling; (D) six-month follow-up angiogram demonstrating significant recanalization of the aneurysm; (E) Virtual stenting (Siemens, Forchheim, Germany) of the planned flow diversion across the aneurysm neck and $(\mathbf{F})$ the actual, eventual treatment using flow diversion across the aneurysm's parent vessel; (G) six-month post-operative angiogram after flow diversion demonstrating Raymond grade I occlusion of the left PCA aneurysm. PCA = posterior cerebral artery.
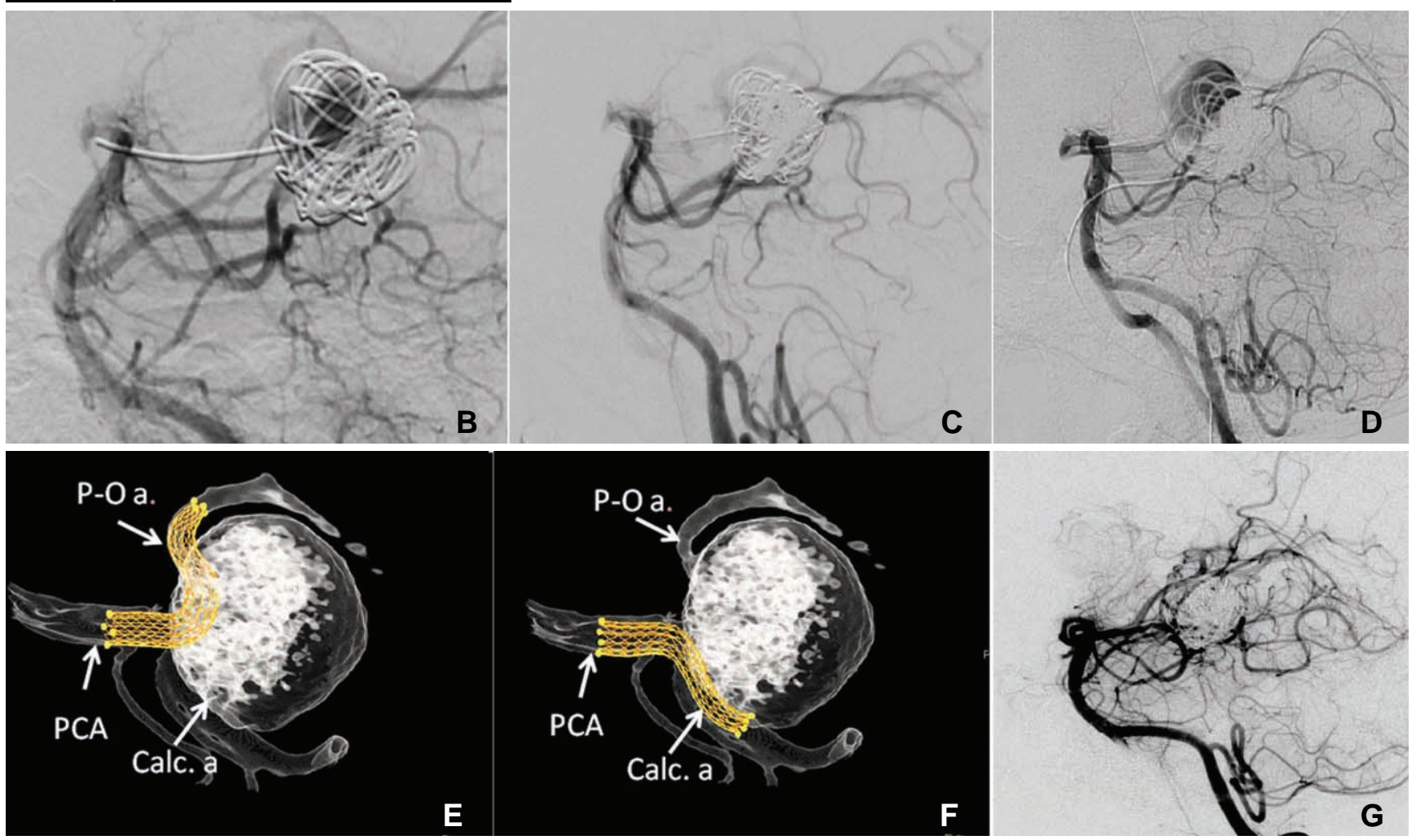


\section{Progressive Deconstruction of a Distal PCA Aneurysm}

procedure, and it revealed significant recanalization of the aneurysm. After in-depth discussion with the patient and careful consideration of the local anatomy, a decision was made to use a flow diverter to treat the very large, recanalized aneurysm. The initial plan was to place the flow diverter from the left parieto-occipital artery to the main PCA trunk (Fig. 1C).

The patient was brought to the angiography suite and underwent general endotracheal anesthesia. Through a 6-French sheath, a $115 \mathrm{~cm}$ long Navien 0.072 inch catheter (Covidien, Sunnyvale, CA, USA) was placed in the left vertebral artery. Activated clotting time was maintained over 250 with heparinization throughout the procedure, and aspirin and clopidogrel assays were checked with results showing they were both therapeutic. Under a road mapping technique, a Marksman (Covidien, Sunnyvale, CA, USA) microcatheter was navigated into the left PCA and into the aneurysm.

Initial attempts to catheterize the parieto-occipital artery (superior terminal branch of PCA) were unsuccessful given the small caliber of the vessel, the sharp angle of its takeoff, and the large void of the residual aneurysm. Thus the flow diverter could not be placed across the aneurysm neck as originally planned. After careful consideration, a decision was made to place a flow diverter from the calcarine artery (inferior terminal branch of PCA) to the proximal PCA, thus diverting flow away from the parieto-occipital artery and the aneurysm (Fig. 1D). The left calcarine artery was selectively catheterized with a $2.8 \mathrm{~F} / 3.2 \mathrm{~F} 0.027$ " Marksman microcatheter, and a $2.5 \times 10 \mathrm{~mm}$ Pipeline Embolization Device (PED, Covidien, Sunnyvale, CA, USA) was placed from the calcarine artery to the PCA trunk. At this time, a cerebral angiogram was performed which demonstrated patency of both calcarine artery and parieto-occipital artery with increased stagnation of blood flow into the aneurysm. The patient tolerated the procedure well. The patient did not have any new neurological deficit after PED placement and post-procedure MRI did not demonstrate stroke in the territory of the calcarine and parieto-occipital arteries. She was discharged home on daily aspirin and clopidogrel.

A diagnostic cerebral angiography at 6 months after the PED placement demonstrated complete Raymond grade 1 occlusion of the aneurysm (Fig. 2). Unlike the cited progressive deconstruction case [7], there was not significant vessel collateralization. She remained neurologically intact.

\section{DISCUSSION}

Wajnberg et al. reported an initial case of progressive deconstruction for a distal giant middle cerebral artery (MCA) aneurysm [3]. In that case, a Pipeline Embolization Device could not be placed within the parent vessel to cover the aneurysm, so the Pipeline was subsequently placed across the aneurysm parent vessel from a separate MCA trunk into the proximal MCA. Follow up angiography revealed occlusion of the aneurysm and parent vessel. There was also extensive collateralization of the remaining internal carotid and external carotid arteries to supply the territory of brain originally supplied by the aneurysm's parent vessel.

In the present case, a similar technical challenge prevented flow diversion across a distal PCA aneurysm neck. The decision was made to place a Pipeline Embolization Device across the parieto-occipital artery, which hosted the aneurysm. Follow-up angiography revealed aneurysm occlusion, but did not show the same degree of collateralization seen in the prior report by Wajnberg. The parent vessel in our case probably had better initial collateralization and a smaller vascular territory than the MCA trunk in their report. In both of these cases of very challenging aneurysms, clinical and angiographic outcomes were excellent.

Parent vessel occlusion for aneurysm treatment has provided many satisfactory outcomes when reconstructive strategies were not feasible. Hallacq et al. treated 10 P2-segment aneurysms with parent vessel occlusion and reported no neurological complications [4]. Roh et al. had infarcts in three of seven patients treated with parent vessel occlusion, but only one patient was symptomatic with transient ischemia [5]. Liu et al. treated 12 non-saccular PCA aneurysms with parent vessel occlusion with no permanent neurological deficits [6]. Kashiwazaki et al. treated nine PCA aneurysms with parent vessel occlusion, resulting in two cases of non-disabling morbidity; however, seven out of nine had evidence of small infarction [7].

In the presented case, parent vessel occlusion may have been a successful strategy. However, in contrast to the instantaneous deconstruction of vessel occlusion, progressive deconstruction using flow diversion away from the parent vessel may decrease the risk of infarction. Flow diverters are low porosity stents. The small cells decrease flow but remain patent when a pressure gradient exists across them. With progressive deconstruction, the flow and pressure gradient across the 


\section{Andrew K. Johnson, et al.}

flow diverter presumably decrease as collateralization occurs. This process thus prevents periods of severe ischemia in the parent vessel territory. In conclusion, a strategy of progressive deconstruction with competitive flow diversion away from the parent vessel may be superior to parent vessel occlusion when reconstructive strategies are not feasible.

\section{CONCLUSION}

The progressive deconstruction technique may occlude both aneurysm and parent vessel in a timeframe, which allows for adequate vascular collateral formation without infarction. When considering parent vessel occlusion, flow diversion away from the aneurysm-hosting artery may cure the aneurysm with a decreased risk of infarction. There is extremely limited experience with progressive deconstruction, and standard open and endovascular aneurysm treatments should always be considered first.

\section{References}

1. Wu A, Chang SW, Deshmukh P, Spetzler RF, Preul MC. Through the choroidal fissure: a quantitative anatomic comparison of 2 incisions and trajectories (transsylvian transchoroidal and lateral transtemporal). Neurosurgery 2010;66(6 Suppl Operative): 221228; discussion 228-229

2. Kocaeli H, Chaalala C, Abruzzo TA, Zuccarello M. Results of surgical management for posterior cerebral artery aneurysms: 7year experience in the endovascular era. Acta Neurochir (Wien) 2009; 151:1583-1591

3. Wajnberg E, Silva TS, Johnson AK, Lopes DK. Progressive deconstruction: a novel aneurysm treatment using the pipeline embolization device for competitive flow diversion: case report. Neurosurgery 2014;10(Suppl 1):E161-166; discussion E166

4. Hallacq P, Piotin M, Moret J. Endovascular occlusion of the posterior cerebral artery for the treatment of $\mathrm{p} 2$ segment aneurysms: retrospective review of a 10-year series. AJNR Am J Neuroradiol 2002;23:1128-1136

5. Roh HG, Kim SS, Han H, Kang HS, Moon WJ, Byun HS. Endovascular treatment of posterior cerebral artery aneurysms using detachable coils. Neuroradiology 2008;50:237-242

6. Liu L, He H, Jiang C, Lv X, Li Y. Deliberate parent artery occlusion for non-saccular posterior cerebral artery aneurysms. Interv Neuroradiol 2011;17:159-168

7. Kashiwazaki D, Ushikoshi S, Asano T, Osanai T, Kuroda S, Houkin K. Endovascular treatment for aneurysms of the posterior cerebral artery: 12 years' experience with 21 cases. Acta Neurochir (Wien) 2011;153:2151-2158 